G. Hajdu · L. Hajdu

\title{
Hosszú's equation over the Gaussian and Eisenstein integers
}

Received: date / Accepted: date

\begin{abstract}
We consider Hosszú's famous functional equation $f(x)+f(y)=$ $f(x y)+f(x+y-x y)$. We completely describe the set of functions $f: R \rightarrow \mathbb{A}$ satisfying this equation, where $R$ is the set of the Gaussian or Eisenstein integers and $\mathbb{A}$ is an arbitrary Abelian group.
\end{abstract}

Keywords Hosszú's functional equation, functional equations

Mathematics Subject Classification (2000) 39B52

\section{Introduction}

In 1967 Miklós Hosszú introduced the following functional equation and asked for its solutions:

$$
f(x)+f(y)=f(x y)+f(x+y-x y) .
$$

Research supported in part by the János Bolyai Research Fellowship of the Hungarian Academy of Sciences, by the NKTH and by the OTKA grants T043080, T042985 and T048791.

G. Hajdu

Institute of Mathematics and Computer Science

Faculty of Mechanical Engineering

Szent István University

H-2103 Gödöllő, Páter K. u. 1., Hungary

E-mail: godenyne.hajdu.gabriella@gek.szie.hu

L. Hajdu

Institute of Mathematics, University of Debrecen, and the Number Theory Research Group

of the Hungarian Academy of Sciences,

H-4010 Debrecen, P.O.B. 12, Hungary

E-mail: hajdul@math.klte.hu 
It is said that one of his purposes in doing so was to annoy mathematicians dealing with functional equations, since this equation resists all the usual substitution tricks. Hosszú himself could describe the solutions $f: \mathbb{R} \rightarrow \mathbb{R}$ under the assumption that $f$ is differentiable. Since then many authors have considered this equation, and the literature of Hosszú's equation has become quite extensive. We restrict our attention to results where the complete solution set is described, for some choice of the domain and range of the functions $f$ involved. We write $\mathcal{H}(A, B)$ for the set of solutions of (1) with $D_{f}=A$ and $R_{f} \subset B$. Further, throughout the paper $\mathbb{A}$ denotes an arbitrary Abelian group.

The sets $\mathcal{H}(\mathbb{R}, \mathbb{A})$ and $\mathcal{H}(\mathbb{C}, \mathbb{A})$ were determined independently by Blanuša [1] and Daróczy [2]. Światak [8] described $\mathcal{H}(K, \mathbb{A})$ under certain assumptions on $\mathbb{A}$, where $K$ is a field of characteristic different from 2 and 3 . Davison in [3] and [4] characterized the sets $\mathcal{H}(\mathbb{Q}, \mathbb{A})$ and $\mathcal{H}(\mathbb{Z} / p \mathbb{Z}, \mathbb{A})(p \geq 5$ is a prime), and $\mathcal{H}(K, \mathbb{A})$, respectively. In the latter case $K$ is a field with at least five elements. Note that in all these cases the solutions to (1) were all affine, i.e. $f(x+y)+f(0)=f(x)+f(y)$ holds for every $x, y \in D_{f}$.

There are also results in the case when $D_{f}$ is only a ring. Davison and Redlin [5] proved that every $f \in \mathcal{H}(R, \mathbb{A})$ is affine, where $R$ is the ring generated by a primitive $m$-th root of unity such that $m$ is odd and not a power of 3. Davison [3] described all solutions in $\mathcal{H}(\mathbb{Z}, \mathbb{A})$, as well. Interestingly, in this case beside the affine solutions there are non-affine ones, too. Note that Głowacki and Kuczma [6] gave a different proof for this result, under certain mild conditions on $\mathbb{A}$.

In this paper we solve equation (1) under the assumption that the domain of the function $f$ is the ring of Gaussian integers $\mathbb{G}$ or Eisenstein integers $\mathbb{E}$, with $R_{f} \subset \mathbb{A}$ in both cases. Our results may be considered as a continuation of those of Davison [3] obtained over $\mathbb{Z}$. In particular, beside affine solutions we find non-affine ones, as well. We note that both $\mathbb{G}$ and $\mathbb{E}$ are generated by roots of unity, however, these rings are excluded from the above mentioned result of Davison and Redlin [5]. As we obtain non-affine solutions as well, our results also show that the assumptions on $m$ in [5] are necessary, at least they cannot be simply removed.

The structure of this paper is as follows. In the next section we give our results, and we also give a kind of explanation why we restrict our attention only to the rings $\mathbb{G}$ and $\mathbb{E}$. In Section 3 we give the proofs of our theorems.

\section{Results}

In this section we state two theorems which give the complete solutions of Hosszú's equation over the Gaussian and the Eisenstein integers, respectively. In both cases the solutions map into an arbitrary Abelian group $\mathbb{A}$.

Let $\mathbb{G}$ denote the ring of the Gaussian integers, that is,

$$
\mathbb{G}=\mathbb{Z}[i]=\{a+b i: a, b \in \mathbb{Z}\} .
$$


Theorem 1 Suppose that $f: \mathbb{G} \rightarrow \mathbb{A}$ is a solution to (1). Then $f$ can be written as $f=\sum_{j=1}^{5} \alpha_{j} f_{j}$ where $\alpha_{j} \in \mathbb{A}(j=1, \ldots, 5)$ with $2 \alpha_{5}=0$, and the functions $f_{j}: \mathbb{G} \rightarrow \mathbb{Z}(j=1, \ldots, 4)$ and $f_{5}: \mathbb{G} \rightarrow \mathbb{Z} / 2 \mathbb{Z}$ are given by

$$
\begin{aligned}
& f_{1}(z)=1, \quad f_{2}(z)=\Re(z), \quad f_{3}(z)=\Im(z), \\
& f_{4}(z)=\Re(t) \text { where } z=(1+i) t+\delta(\delta \in\{0,1\}), \\
& f_{5}(z)= \begin{cases}1, & \text { if } z \equiv 1+i \quad(\bmod 2), \\
0, & \text { otherwise. }\end{cases}
\end{aligned}
$$

Note that $\mathbb{G}$ is a Euclidean ring. Moreover, $1+i$ is a prime in $\mathbb{G}$ with norm 2 over $\mathbb{Q}$, so $f_{4}$ in Theorem 1 is well-defined. We also mention that we have $f_{4}(z)=\left[\frac{\Re(z)+\Im(z)}{2}\right]$ for all $z \in \mathbb{G}$.

In view of the explicit forms of the functions $f_{1}, \ldots, f_{5}$ we have the following straightforward consequence of Theorem 1 .

Corollary 1 For any solution $f: \mathbb{G} \rightarrow \mathbb{A}$ to (1) we have

$$
f(x+y)+f(0)=f(x)+f(y) \text { for all } x, y \in \mathbb{G} \text { with } 2 \mid x y .
$$

In the next result we describe the solutions to (1) over the Eisenstein integers $\mathbb{E}$. As is well known (see e.g. [9] p. 72) this ring is given by

$$
\mathbb{E}=\mathbb{Z}[\xi]=\{a+b \xi: a, b \in \mathbb{Z}\}, \text { where } \xi=\frac{-1+i \sqrt{3}}{2} .
$$

In other words, each $z \in \mathbb{E}$ can be uniquely written as $z=z_{1}+z_{2} \xi$ with some $z_{1}, z_{2} \in \mathbb{Z}$. By the coordinates of $z$ we shall mean $(z)_{1}=z_{1}$ and $(z)_{2}=z_{2}$, and we use this notation whenever we work in $\mathbb{E}$.

Theorem 2 Suppose that $f: \mathbb{E} \rightarrow \mathbb{A}$ is a solution to (1). Then $f$ is of the form $f=\sum_{j=1}^{5} \alpha_{j} f_{j}$ where $\alpha_{j} \in \mathbb{A}$, and the functions $f_{j}: \mathbb{E} \rightarrow \mathbb{Z}(j=1, \ldots, 5)$ are given by

$$
\begin{aligned}
& f_{1}(z)=1, \quad f_{2}(z)=(z)_{1}, \quad f_{3}(z)=(z)_{2}, \\
& f_{4}(z)=\left\{\begin{array}{lll}
(t)_{1}, & \text { if } \quad z=(1+2 \xi) t+\delta \\
(t)_{1}-1, & \text { if } \quad z=(1+2 \xi) t+1,
\end{array} \quad(\delta \in\{0,1+\xi\}),\right. \\
& f_{5}(z)=\left\{\begin{array}{lll}
(t)_{2}, & \text { if } \quad z=2 t, \\
(t)_{2}+1, & \text { if } \quad z=2 t+\delta \quad(\delta \in\{1, \xi, 1+\xi\}) .
\end{array}\right.
\end{aligned}
$$

We mention that $\mathbb{E}$ is also a Euclidean ring. Further, the norms of $1+2 \xi$ and 2 are 3 and 4 over $\mathbb{Q}$, respectively. Hence the functions $f_{4}$ and $f_{5}$ in Theorem 2 are well-defined. Further, we also have $f_{4}(z)=\left[\frac{2(z)_{2}-(z)_{1}}{3}\right]$ and $f_{5}(z)=\left[\frac{(z)_{2}-(z)_{1}+1}{2}\right]+\left[\frac{(z)_{1}+1}{2}\right]$ for all $z \in \mathbb{E}$.

Again, knowing the explicit form of the solutions $f: \mathbb{E} \rightarrow \mathbb{A}$ to (1) we can formulate a simple corollary of the above statement. Namely, we have the following straightforward consequence of Theorem 2. 
Corollary 2 For any solution $f: \mathbb{E} \rightarrow \mathbb{A}$ to (1) we have

$$
f(x+y)+f(0)=f(x)+f(y) \text { for all } x, y \in \mathbb{E} \text { with } 2+4 \xi \mid x y .
$$

\subsection{Limitations of the method}

In this subsection we briefly explain why we restrict our attention to the rings $\mathbb{G}$ and $\mathbb{E}$.

Following the method of [3], we proceed by a kind of induction. We show that if $f$ is a solution to (1), then the values of $f$ taken on "sufficiently large" arguments, can be obtained by the values of $f$ taken on "smaller" ones. However, to make this principle work we need several assumptions. First, it is natural and convenient to consider the ring of integers $R$ of some algebraic number field. In this case we are automatically equipped with the usual absolute value. Further, $R$ should be discrete, i.e. for any positive real $r$, there should be only finitely many $z \in R$ with $|z|<r$ - otherwise the "induction step" fails. At this point we mention that to solve a somewhat related problem of Ramanujan over certain finitely generated domains, the authors in [7] used another norm, which has the "discrete" property for the ring of integers of any algebraic number field. However, in case of Hosszú's equation (at least in the present method) we need some kind of norm which is multiplicative on $R$ (in particular, we cannot use the $L_{\infty}$-like norm from [7]). So it seems that in this case we need to stick to the ordinary absolute value.

Finally, observe that we have $x+y-x y=(x-1)(1-y)+1$. This means that the "large" arguments in (1) (i.e. $x y$ and $x+y-x y$ ) are of the form "product" and "product plus one", respectively. Hence if we have an element $p$ in $R$ of norm 2 over $\mathbb{Q}$ (such as 2 in $\mathbb{Z}$ and $1+i$ in $\mathbb{G}$ ), then any $z \in R$ can be written as one of these "large" arguments in a non-trivial way. Otherwise, lacking such a $p$, we do not have this possibility. As there is no such $p \in \mathbb{E}$, already in this case we need some additional reasoning. Fortunately, in $\mathbb{E}$ there are roots of unity, and by their help we can reach the appropriate residue classes modulo $1+2 \xi=i \sqrt{3}$. (Note that in the ring $\mathbb{Z}[i \sqrt{3}]$ the method would fail in its present form.)

However, the assumption that $R$ should be discrete, already restricts us (beside $\mathbb{Z}$ ) to the rings of integers of imaginary quadratic fields. As the only such ring containing an element of norm 2 is $\mathbb{G}$, and the only such ring (apart from $\mathbb{G}$ ) containing roots of unity is $\mathbb{E}$, we arrive at these rings in a natural way. Summarizing these facts, we conclude that to extend this "inductive" method to other rings, probably some extra tools are needed.

\section{Proofs}

In this section we give the proofs of our results. 
3.1 The proof of Theorem 1

Put $I_{\mathbb{G}}=\{-1,0,1, i, 1+i\}$. As it turns out, the set $I_{\mathbb{G}}$ plays a crucial role in the proof of this theorem. Further, we need the following two lemmas.

Lemma 1 Let $f: \mathbb{G} \rightarrow \mathbb{A}$ be a solution to (1). Then there exist $\alpha_{j} \in \mathbb{A}$ $(j=1, \ldots, 5)$ with $2 \alpha_{5}=0$ such that $f(z)=\sum_{j=1}^{5} \alpha_{j} f_{j}(z)$ for all $z \in I_{\mathbb{G}}$. Here the functions $f_{j}(j=1, \ldots, 5)$ are defined in Theorem 1 .

Proof Substitute the values $-1,0,1, i, 1+i$ into $f$ and solve the implied system of linear equations for $\alpha_{j}(j=1, \ldots, 5)$. Since the determinant of the matrix of the system is 1 , this can be done in $\mathbb{A}$ without any problem. We get that the unique solution is given by

$$
\begin{aligned}
& \alpha_{1}=f(0), \\
& \alpha_{2}=-f(0)+f(1), \\
& \alpha_{3}=-f(0)+f(i), \\
& \alpha_{4}=-f(-1)+2 f(0)-f(1), \\
& \alpha_{5}=f(-1)-f(0)-f(i)+f(1+i) .
\end{aligned}
$$

It remains to show that $2 \alpha_{5}=0$. Substituting appropriate values for $x$ and $y$ in (1) we successively get the following identities:

$$
\begin{aligned}
f(-i) & =f(1)+f(-1)-f(i)(x=i, y=-i), \\
f(1-i) & =f(1+i)+f(1)+f(-1)-2 f(i)(x=i, y=1-i), \\
f(2) & =2 f(1+i)+f(1)+f(-1)-2 f(i)-f(0)(x=1+i, y=1-i), \\
f(2-2 i) & =2 f(1+i)+2 f(1)+2 f(-1)-4 f(i)-f(0)(x=2, y=1-i),
\end{aligned}
$$

and

$$
\begin{aligned}
f(1+2 i) & =2 f(i)-f(-1)(x=i, y=i), \\
f(-1-i) & =f(1+i)+2 f(-1)-2 f(i)(x=-1, y=1+i) .
\end{aligned}
$$

Hence we deduce

$$
f(4)=2 f(1+i)+2 f(1)-2 f(i)-f(0)(x=1+i, y=2-2 i) .
$$

Substituting $x=y=2$ in (1) we also have $f(4)=2 f(2)-f(0)$. Combining the last two equalities we obtain

$$
2(f(-1)-f(0)-f(i)+f(1+i))=2 \alpha_{5}=0,
$$

and the lemma follows.

Remark 1 From the proof of the above lemma it is clear that the functions $f_{j}(j=1, \ldots, 5)$ are linearly independent over $\mathbb{A}$ in general, that is, it is not possible to replace $f_{j}(j=1, \ldots, 5)$ in Theorem 1 by a smaller set.

The next lemma implies that the values taken by a solution $f$ on the set $I_{\mathbb{G}}$ determine $f$ uniquely. 
Lemma 2 Let $f: \mathbb{G} \rightarrow \mathbb{A}$ be a solution to (1) satisfying $f(z)=0$ for all $z \in I_{\mathbb{G}}$. Then $f(z)=0$ for all $z \in \mathbb{G}$.

Proof First we show that if a solution $f$ vanishes on the set

$$
I_{0}:=\{w \in \mathbb{G}:|w| \leq 2+2 \sqrt{2}\}
$$

then $f$ vanishes on the whole $\mathbb{G}$. (Note that $I_{0} \supset I_{\mathbb{G}}$.) We present an inductive argument. Let $z \in \mathbb{G} \backslash I_{0}$, and assume that $f(w)=0$ whenever $|w|<|z|$. We distinguish two cases.

Assume first that $z \equiv 0(\bmod 1+i)$, i.e. $z=(1+i) t$ for some $t \in \mathbb{G}$. Putting $x=1+i$ and $y=t$ we have $x y=z$ and $x+y-x y=i(1-t)+1$. Moreover, as $|z|>2+2 \sqrt{2}$ a simple calculation gives

$$
|x+y-x y|=\left|\frac{2 i-i z}{1+i}\right| \leq \frac{1}{\sqrt{2}}|z|+\sqrt{2}<|z| .
$$

Since $|x|<|z|$ and $|y|<|z|$ are also valid, we obtain that $f(z)=f(x y)=0$ in this case.

Suppose next that $z \equiv 1(\bmod 1+i)$ that is $z=(1+i) t+1$ for some $t \in \mathbb{G}$. Writing $x=i$ and $y=i t+1$, we get $x+y-x y=z$ and $x y=i-t$. Further, using $|z|>2+2 \sqrt{2}$ one can easily derive that

$$
|x y|=|y|=\left|\frac{i z+1}{1+i}\right| \leq \frac{|z|+1}{\sqrt{2}}<|z| \text { and }|x|<|z|
$$

hold. This yields again $f(z)=f(x+y-x y)=0$.

Note that as the norm of $1+i$ (as an element of the number field $\mathbb{Q}(i)$ over $\mathbb{Q}$ ) is 2 , there are no other cases to distinguish. Further, since for any $z \in \mathbb{G}$ there are only finitely many Gaussian integers that are shorter than $z$, we get that if $f$ vanishes on $I_{0}$ then $f$ vanishes on the whole $\mathbb{G}$.

It only remains to show that $f \equiv 0$ on $I_{\mathbb{G}}$ implies $f \equiv 0$ on $I_{0}$. This can be done by a simple calculation. We do not give excessive details, just briefly outline a simple algorithm that we used to verify the statement.

As the initialization step, put $S=I_{\mathbb{G}}$. Then find some "short" $x, y \in \mathbb{G}$ such that exactly three out of the four arguments $x y, x y x+y-x y$ in (1) belong to $S$. Then add the fourth argument to $S$, and repeat the whole process. Continue this procedure until $S \supset I_{0}$. For example, in the first loop take $x=i$ and $y=-i$, whence $x y=1$ and $x+y-x y=-1$. Thus we can add $-i$ to $S$. In the next loop choose $x=i$ and $y=1-i$, yielding $x y=1+i$ and $x+y-x y=-i$. So we can add $1-i$ to $S$, etc.

As the result of this procedure after a few loops (with "short" values for $x$ and $y$ ) we get some $S$ with $S \supset I_{0}$. Thus we conclude that $f \equiv 0$ also on $I_{0}$, and the lemma follows.

Now we are prepared to give the

Proof (of Theorem 1) It is obvious that the functions $f_{1}, f_{2}, f_{3}$ are solutions to $(1)$, and one can easily check that $f_{5}$ is also a solution. Hence we deal only with $f_{4}$. Let $x$ and $y$ be arbitrary elements of $\mathbb{G}$, and put

$$
x=(1+i) t_{1}+\delta_{1}, \quad y=(1+i) t_{2}+\delta_{2}
$$


with $t_{1}, t_{2} \in \mathbb{G}$ and $\delta_{1}, \delta_{2} \in\{0,1\}$. Then we have

$$
x y=(1+i)\left((1+i) t_{1} t_{2}+\delta_{1} t_{2}+\delta_{2} t_{1}\right)+\delta_{1} \delta_{2}
$$

and

$$
x+y-x y=(1+i)\left(t_{1}+t_{2}-(1+i) t_{1} t_{2}-\delta_{1} t_{2}-\delta_{2} t_{1}\right)+\delta_{1}+\delta_{2}-\delta_{1} \delta_{2} .
$$

Observe that $\delta_{1}=\delta_{1} \delta_{2}$ and $\delta_{2}=\delta_{1}+\delta_{2}-\delta_{1} \delta_{2}$, or vica versa. Hence, as $\Re(u+v)=\Re(u)+\Re(v)$ for all $u, v \in \mathbb{G}$, we get that $f_{4}$ is also a solution to (1). Thus for any coefficients $\alpha_{j} \in \mathbb{A}(j=1, \ldots, 5)$ with $2 \alpha_{5}=0$ the function $\sum_{j=1}^{5} \alpha_{j} f_{j}: \mathbb{G} \rightarrow \mathbb{A}$ is a solution to $(1)$.

Now we only have two show that any solution to (1) can be written as a linear combination of the functions $f_{j}(j=1, \ldots, 5)$ over $\mathbb{A}$, with $2 \alpha_{5}=0$. Suppose that $f: \mathbb{G} \rightarrow \mathbb{A}$ satisfies (1). Then by Lemma 1 there exist $\alpha_{j} \in \mathbb{A}$ $(j=1, \ldots, 5)$ with $2 \alpha_{5}=0$ such that $g:=f-\sum_{j=1}^{5} \alpha_{j} f_{j}$ vanishes on $I_{\mathbb{G}}$. As $g: \mathbb{G} \rightarrow \mathbb{A}$ is a solution to (1), by Lemma 2 we obtain that $g \equiv 0$ on $\mathbb{G}$. This proves the theorem.

\subsection{The proof of Theorem 2}

The proof of Theorem 2 is similar to that of Theorem 1, up to one important point. Namely, $\mathbb{E}$ does not contain elements of norm 2 over $\mathbb{Q}$. Hence we cannot claim that for some $\beta \in \mathbb{E}$ each $\gamma \in \mathbb{E}$ is congruent to 0 or 1 modulo $\beta$. In other words, we cannot utilize directly the form of the arguments $x y$ and $x+y-x y=(x-1)(1-y)+1$ of $f$. However, we can make use of the roots of unity in $\mathbb{E}$, to "switch" between the residue classes of $1+2 \xi=i \sqrt{3}$.

Put $I_{\mathbb{E}}=\{-1,0,1, \xi, 1+\xi\}$; this set will play a role similar to $I_{\mathbb{G}}$ in the proof of Theorem 1 . The proof of Theorem 2 relies on the following two lemmas.

Lemma 3 Let $f: \mathbb{E} \rightarrow \mathbb{A}$ be a solution to (1). Then there exist $\alpha_{j} \in \mathbb{A}$ $(j=1, \ldots, 5)$ such that $f(z)=\sum_{j=1}^{5} \alpha_{j} f_{j}(z)$ for all $z \in I_{\mathbb{E}}$, where the functions $f_{j}(j=1, \ldots, 5)$ are defined in Theorem 2.

Proof Substitute the values $-1,0,1, \xi, 1+\xi$ into $f$ and solve the implied system of linear equations for $\alpha_{j}(j=1, \ldots, 5)$. Since the determinant of the matrix of the system is 1 , this can be done in $\mathbb{A}$ without any problem. We get that the unique solution is given by

$$
\begin{aligned}
& \alpha_{1}=f(0), \\
& \alpha_{2}=-f(\xi)+f(1+\xi), \\
& \alpha_{3}=-f(-1)+2 f(\xi)-f(1+\xi), \\
& \alpha_{4}=f(-1)-f(1)-2 f(\xi)+2 f(1+\xi), \\
& \alpha_{5}=f(-1)-f(0)-f(\xi)+f(1+\xi),
\end{aligned}
$$

and the lemma follows. 
Remark 2 As in case of $\mathbb{G}$, in view of the proof of the above lemma we obtain that the functions $f_{j}: \mathbb{E} \rightarrow \mathbb{A}(j=1, \ldots, 5)$ are linearly independent over $\mathbb{A}$ in general, so they cannot be replaced by fewer functions in Theorem 2 .

The following lemma yields that the values taken by a solution $f$ on $I_{\mathbb{E}}$ determine $f$ uniquely.

Lemma 4 Let $f$ be a solution to (1) satisfying $f(z)=0$ for all $z \in I_{\mathbb{E}}$. Then $f(z)=0$ for all $z \in \mathbb{E}$.

Proof Like in the proof of Lemma 2, first we prove that a bounded set has the desired property, that is, if $z \in \mathbb{E}$ is "long enough" then $f(z)=0$ holds. More precisely, we show that if a solution $f: \mathbb{E} \rightarrow \mathbb{A}$ to (1) is identically zero on the set

$$
I_{0}:=\{w \in \mathbb{E}:|w| \leq 2 \sqrt{3}+2\},
$$

then $f$ is identically zero on $\mathbb{E}$. (Note that $I_{0} \supset I_{\mathbb{E}}$.) We present an inductive argument also in this case. Let $z \in \mathbb{E} \backslash I_{0}$, and assume that $f(w)=0$ for all $|w|<|z|$. We distinguish three cases, according to the residue classes of $1+2 \xi$

Assume first that $z \equiv 0(\bmod 1+2 \xi)$. Then as $\xi$ is a unit in $\mathbb{E}$ we can write $z=-\xi(1+2 \xi) t$ for some $t \in \mathbb{E}$. Letting $x=t$ and $y=-\xi(1+2 \xi)$, we have $x y=z$ and $x+y-x y=\xi^{-1} t-\xi^{-1}+1$. Note that $\xi^{-1}=-1-\xi=\frac{-1-i \sqrt{3}}{2}$. As $|z|>2 \sqrt{3}+2$, a simple calculation gives

$$
|x+y-x y|=\left|\frac{-\xi z}{1+2 \xi}-\xi^{-1}+1\right| \leq \frac{|z|}{\sqrt{3}}+\sqrt{3}<|z| .
$$

As we also have $|x|<|z|$ and $|y|<|z|$, we get that $f(z)=f(x y)=0$ in this case.

Suppose next that $z \equiv 1(\bmod 1+2 \xi)$. Then we have $z=\xi(1+2 \xi) t+1$ for some $t \in \mathbb{E}$. Setting $x=1-t$ and $y=\xi(1+2 \xi)+1=\xi^{-1}$, we obtain $x+y-x y=z$ and $x y=(1-t) \xi^{-1}$. Now we get

$$
|x y|=|x|=\left|\frac{-z+\xi^{-1}}{\xi(1+2 \xi)}\right| \leq \frac{|z|+1}{\sqrt{3}}<|z| \quad \text { and } \quad|y|<|z| .
$$

This implies that $f(z)=f(x+y-x y)=0$ also in this case.

Finally, assume that $z \equiv 1+\xi(\bmod 1+2 \xi)$. Put $x=z$ and $y=-\xi$. Then $x y=-\xi z$ and in particular, $x y \equiv 1(\bmod 1+2 \xi)$. Hence by $|x y|=$ $|z|$ the assertions in the previous paragraph yield $f(x y)=0$. Further, we have $x+y-x y=z(1+\xi)-\xi$, whence $x+y-x y \equiv 0(\bmod 1+2 \xi)$ and $|x+y-x y| \leq|z|+1$. Applying now the first part of the proof (with $z \equiv 0$ $(\bmod 1+2 \xi))$ writing $x+y-x y$ in place of $z$ and using our assumption on $|z|$, a simple calculation shows that $f(x+y-x y)=0$ is also valid. Hence, as $|y|<|z|$, we get that $f(z)=f(x)=0$ also in this case.

Now it remains only to show that $f \equiv 0$ on $I_{0}$, provided that $f \equiv 0$ on $I_{\mathbb{E}}$. The proof of this assertion can be done by a procedure as in Lemma 2 , and we omit the details. The proof of Lemma 4 is complete. 
Now we can give the

Proof (of Theorem 2) It is obvious that the functions $f_{1}, f_{2}, f_{3}$ are solutions to (1). To prove that the function $f_{4}$ is also a solution let $x, y \in \mathbb{E}$ be arbitrary, and put

$$
x=(1+2 \xi) t_{1}+\delta_{1}, \quad y=(1+2 \xi) t_{2}+\delta_{2}
$$

with $t_{1}, t_{2} \in \mathbb{E}$ and $\delta_{1}, \delta_{2} \in\{0,1,1+\xi\}$. Then we have

$$
x y=(1+2 \xi)\left((1+2 \xi) t_{1} t_{2}+\delta_{1} t_{2}+\delta_{2} t_{1}\right)+\delta_{1} \delta_{2},
$$

$x+y-x y=(1+2 \xi)\left(t_{1}+t_{2}-(1+2 \xi) t_{1} t_{2}-\delta_{1} t_{2}-\delta_{2} t_{1}\right)+\delta_{1}+\delta_{2}-\delta_{1} \delta_{2}$.

If either $\delta_{1}$ or $\delta_{2}$ belongs to $\{0,1\}$ then $\delta_{1}=\delta_{1} \delta_{2}$ and $\delta_{2}=\delta_{1}+\delta_{2}-\delta_{1} \delta_{2}$, or vica versa. Hence, as $(u+v)_{1}=(u)_{1}+(v)_{1}$ for all $u, v \in \mathbb{E}$, we are done in this case. So we are left with the possibility $\delta_{1}=\delta_{2}=1+\xi$. Now we have

$$
f_{4}(x)+f_{4}(y)=\left(t_{1}+t_{2}\right)_{1},
$$

and as $\xi=(1+\xi)(1+2 \xi)+1$ and $2+\xi=-\xi(1+2 \xi)$, also

$$
f_{4}(x y)+f_{4}(x+y-x y)=\left(t_{1}+t_{2}+1\right)_{1}-1 .
$$

Since these expressions are equal, $f_{4}$ is also a solution to (1).

To check that $f_{5}$ is a solution as well, take arbitrary $x, y \in \mathbb{E}$ and set

$$
x=2 t_{1}+\delta_{1}, \quad y=2 t_{2}+\delta_{2}
$$

with $t_{1}, t_{2} \in \mathbb{E}$ and $\delta_{1}, \delta_{2} \in\{0,1, \xi, 1+\xi\}$. Now we have

$$
\begin{gathered}
x y=2\left(2 t_{1} t_{2}+\delta_{1} t_{2}+\delta_{2} t_{1}\right)+\delta_{1} \delta_{2}, \\
x+y-x y=2\left(t_{1}+t_{2}-2 t_{1} t_{2}-\delta_{1} t_{2}-\delta_{2} t_{1}\right)+\delta_{1}+\delta_{2}-\delta_{1} \delta_{2} .
\end{gathered}
$$

If $\delta_{1} \in\{0,1\}$ or $\delta_{2} \in\{0,1\}$ then again $\delta_{1}=\delta_{1} \delta_{2}$ and $\delta_{2}=\delta_{1}+\delta_{2}-\delta_{1} \delta_{2}$, or vica versa, and by $(u+v)_{2}=(u)_{2}+(v)_{2}(u, v \in \mathbb{E})$ we are done. In all the other cases

$$
f_{5}(x)+f_{5}(y)=\left(t_{1}+t_{2}\right)_{2}+2
$$

holds. If $\delta_{1}=\delta_{2}=\xi$, then we have $\delta_{1} \delta_{2}=2(-1-\xi)+1+\xi$ and $\delta_{1}+\delta_{2}-\delta_{1} \delta_{2}=$ $2 \xi+1+\xi$, whence

$$
f_{5}(x y)+f_{5}(x+y-x y)=\left(t_{1}+t_{2}-1\right)_{2}+2,
$$

which shows that $f$ satisfies (1) in this case. When $\delta_{1}=\delta_{2}=1+\xi$ we can write $\delta_{1} \delta_{2}=\xi$ and $\delta_{1}+\delta_{2}-\delta_{1} \delta_{2}=2+\xi$, whence

$$
f_{5}(x y)+f_{5}(x+y-x y)=\left(t_{1}+t_{2}+1\right)_{2}+2,
$$

and (1) is satisfied once again. Finally, by symmetry we may assume that $\delta_{1}=\xi$ and $\delta_{2}=1+\xi$. In this case we get $\delta_{1} \delta_{2}=-2+1$ and $\delta_{1}+\delta_{2}-\delta_{1} \delta_{2}=$ $2(\xi+1)$. Thus

$$
f_{5}(x y)+f_{5}(x+y-x y)=\left(t_{1}+t_{2}+\xi\right)_{2}+1,
$$


and we conclude that $f_{5}$ is a solution to (1). Hence we obtain that for any $\alpha_{j} \in \mathbb{A}(j=1, \ldots, 5)$ the function $\sum_{j=1}^{5} \alpha_{j} f_{j}: \mathbb{E} \rightarrow \mathbb{A}$ is a solution to (1).

Finally, assume that $f: \mathbb{E} \rightarrow \mathbb{A}$ satisfies (1). By Lemma 3 there exist $\alpha_{j} \in \mathbb{A}(j=1, \ldots, 5)$ such that $g:=f-\sum_{j=1}^{5} \alpha_{j} f_{j}$ vanishes on $I_{\mathbb{E}}$. As $g: \mathbb{E} \rightarrow \mathbb{A}$ is a solution to $(1)$, Lemma 4 gives that $g \equiv 0$ on $\mathbb{E}$. Hence the theorem is proved.

\section{Acknowledgments}

We are grateful to the referees for their helpful and useful remarks, in particular for driving our attention to the reference about Eisenstein integers in the book of Narkiewicz [9], and for proposing Corollaries 1 and 2.

\section{References}

[1] D. Blanuša, The functional equation $f(x+y-x y)+f(x y)=f(x)+f(y)$, Aequationes Math. 5 (1970), 63-67.

[2] Z. Daróczy, On the general solution of the functional equation $f(x+y-x y)+$ $f(x y)=f(x)+f(y)$, Aequationes Math. 6 (1971), 130-132.

[3] T. M. K. Davison, On the Functional Equation $f(m+n-m n)+f(m n)=$ $f(m)+f(n)$, Aequationes Math. 10 (1974), 206-211.

[4] T. M. K. Davison, The Complete Solution of Hosszú's Functional Equation over a Field, Aequationes Math. 11 (1974), 273-276.

[5] T. M. K. Davison and L. Redlin, Hosszú's functional equation over rings generated by their units, Aequationes Math. 21 (1980), 121-128.

[6] E. Głowacki and M. Kuczma, Some results on Hosszú's functional equation on integers, Uniw. Śpohlkaski w Katowicach Prace Nauk.-Prace Mat. 9 (1979), $53-63$.

[7] G. Hajdu and L. Hajdu, On an identity of Ramanujan over finitely generated domains, Acta Math. Hungar. 112 (2006), 143-155.

[8] H. Światak, A Proof of the Equivalence of the Equation $f(x+y-x y)+f(x y)=$ $f(x)+f(y)$ and Jensen's Functional Equation, Aequationes Math. 6 (1971), 2429.

[9] W. Narkiewicz, Elementary and Analytic Theory of Algebraic Numbers, PWN, Warsaw, 1974. 